\title{
Un lingakośa inscrit au Musée national du Cambodge (K. 1286)
} Julia Estève, Dominique Soutif

\section{Citer ce document / Cite this document :}

Estève Julia, Soutif Dominique. Un lingakośa inscrit au Musée national du Cambodge (K. 1286). In: Arts asiatiques, tome 69, 2014. pp. 97-106;

doi : https://doi.org/10.3406/arasi.2014.1869

https://www.persee.fr/doc/arasi_0004-3958_2014_num_69_1_1869

Fichier pdf généré le 08/11/2019 


\section{Un lingakośa inscrit au Musée national du Cambodge (K. 1286)}

Le don d'un lingakośa au Musée national du Cambodge en 2009 a constitué un événement majeur pour l'épigraphie et l'histoire de l'art du Cambodge ancien en ce qu'il a doté le pays khmer du premier et seul exemplaire matériel de ce type. Certes, plusieurs modèles avaient été trouvés en pays cam et l'épigraphie khmère faisait référence à ce type d'objets, mais, jusqu'à cette date, aucun objet correspondant n'avait été identifié au Cambodge. En outre, ce lingakośa en particulier présente un intérêt supplémentaire, non seulement parce qu'il porte une inscription mais encore parce qu'il permet d'éclairer une pratique signalée à plusieurs reprises dans l'épigraphie qui demeurait cependant obscure : le don d'un omkkāra ou «syllabe om̆ ».

Son étude est pour nous l'occasion de rappeler, d'une part, les caractéristiques de cette parure de la représentation aniconique du dieu Śiva et, d'autre part, ses occurrences dans l'épigraphie khmère.

Le terme sanskrit kośa/koșa (DSF, s. v., p. 210), «fourreau, gaine, boîte, caisse, enveloppe, trésor, etc. » apparaît très régulièrement dans l'épigraphie khmère. Dans les parties sanskrites, il prend parfois le sens général de «trésor». Il y est également utilisé pour désigner spécifiquement des objets offerts aux divinités et

\footnotetext{
* Nous tenons à remercier ici Chea Socheat du Musée national du Cambodge pour les informations et photographies qu'il nous a communiquées ainsi que Arlo Griffiths (EFEO) pour ses précieuses relectures.
}

ce, de façon réitérative ${ }^{1}$. Il renvoie alors à des objets de nature diverse : un couvercle de kalaśa («flacon, aiguière ») dans l'inscription K. $660^{2}$, un fourreau d'épée en or dans l'inscription K. 713 et des objets parfois difficiles à identifier, comme dans la stance XXX de l'inscription K. 989, dans laquelle une donation de «plus de cent kośa » est faite à Vișnu par Jayavarman V (930 śaka, IC VII, p. 175, 182). Dans certains cas, il est également difficile d'établir à laquelle des deux catégories - sens général de «trésor» ou sens particulier, à déterminer - le terme renvoie ${ }^{3}$.

$\overline{1 . ~ N o u s ~ a v o n s ~ r e l e v e ́ ~ l e s ~ o c c u r r e n c e s ~ d e ~ k o s ́ a ~ o u ̀ ~ c e ~}$ terme est utilisé seul ou en composition pour désigner un objet particulier ou l'entité large de «trésor, magasins royaux» ( $c f$. annexe 1). Il est susceptible d'être utilisé dans d'autres contextes, avec un sens abstrait. Nous avons ainsi ignoré le composé sanskrit manahkośa qui désigne «le palais de l'esprit » (K. 826, st. XXIII ; IC I, p. 21), ains que les cas où kośa est un bhūtasamikhyā dans des chronogrammes (GRIFFITHS 2005, p. 19, n. 29). Nous n'avons pas non plus pris en compte les deux occurrences de mahākoșa relevées par Ph. N. Jenner dans l'inscription K. 258 (face B, l. 37 ; face C, l. 14) car il s'agit, à chaque fois, d'un anthroponyme (DAK, s. v., p. 427). On dénombre ainsi trente-neuf occurrences du terme kośa (ou kosana que George Cœdès considérait comme une autre forme de kośa, cf. IC IV, p. 195, n. 5) présentant un sens concret.

2. Cette identification est confirmée par le parallèle entre le composé sanskrit sakośakalaśa, « un flacon avec sa gaine » et le khmer rūpyakalaśa I haniragaropa ṅan jyan் 8 lin I pāda I, « 1 flacon en argent [avec son] couvercle en hanir pesant 8 jyan், 1 lin, 1 päda» (K. 660, l. 2, 8; 963 śaka; IC I, p. 196, 197, n. 1). Notons que « cover» est l'un des nombreux sens proposés pour kośa par le dictionnaire de M. Monier-Williams (s. v.)

3. C'est par exemple le cas dans la stance VIII de l'inscription K. 55 éditée et traduite par Auguste Barth (589 śaka; ISC, p. 51):

tenāsmiñ giriśe dāyi kośo hutavahadyutih dattakośasahasreṇa sarvvadigkhyātakīrttinā
Pourtant, parmi toutes ces occurrences, la majorité fait clairement référence non seulement à un objet, mais encore à un objet de culte particulier. Une telle identification est, de plus, confirmée par les parties khmères des inscriptions qui, lorsqu'elles utilisent ce terme de kośa, font manifestement référence de façon systématique, à un objet de culte bien précis, qui est fait de métal et qui est spécifiquement lié au rituel śivaïte.

Face à cette polysémie, les chercheurs ont éprouvé des difficultés à préciser ce qu'était, dans le monde matériel, le « kośa » des inscriptions, et ce d'autant plus qu'aucune trouvaille connue ne pouvait lui être directement associée. Son sens fut ainsi très tôt discuté. Abel Bergaigne pensait que cette «gaine» d'un linga devait être identifiée comme étant le « sanctuaire » qui l'accueillait. Ne voyant pas à quoi pouvait correspondre une telle "enveloppe » et doutant des autres sens envisagés, Auguste Barth avait proposé d'y voir le linga luimême ou peut-être sa base (ISCC, p. 252, n. 12). Dans une note additionnelle, il fut le premier à formuler l'hypothèse selon laquelle ce kośa pourrait être comparé à la «châsse richement décorée et faite de lames d'or » qui recouvrait un linga de Prah. Pathom en Thaïlande (ibid., p. 601, n. 1).

«À ce Giriśa, fut donné un trésor brillant comme le feu par ce (prince) qui donnait des trésors par milliers et dont la gloire était proclamée dans toutes les régions. » On peut ici se demander si le premier kośa de cette stance ne désignait pas justement plus spécifiquement un lingakośa de Śiva du type de celui que nous allons présenter dans cet article. 

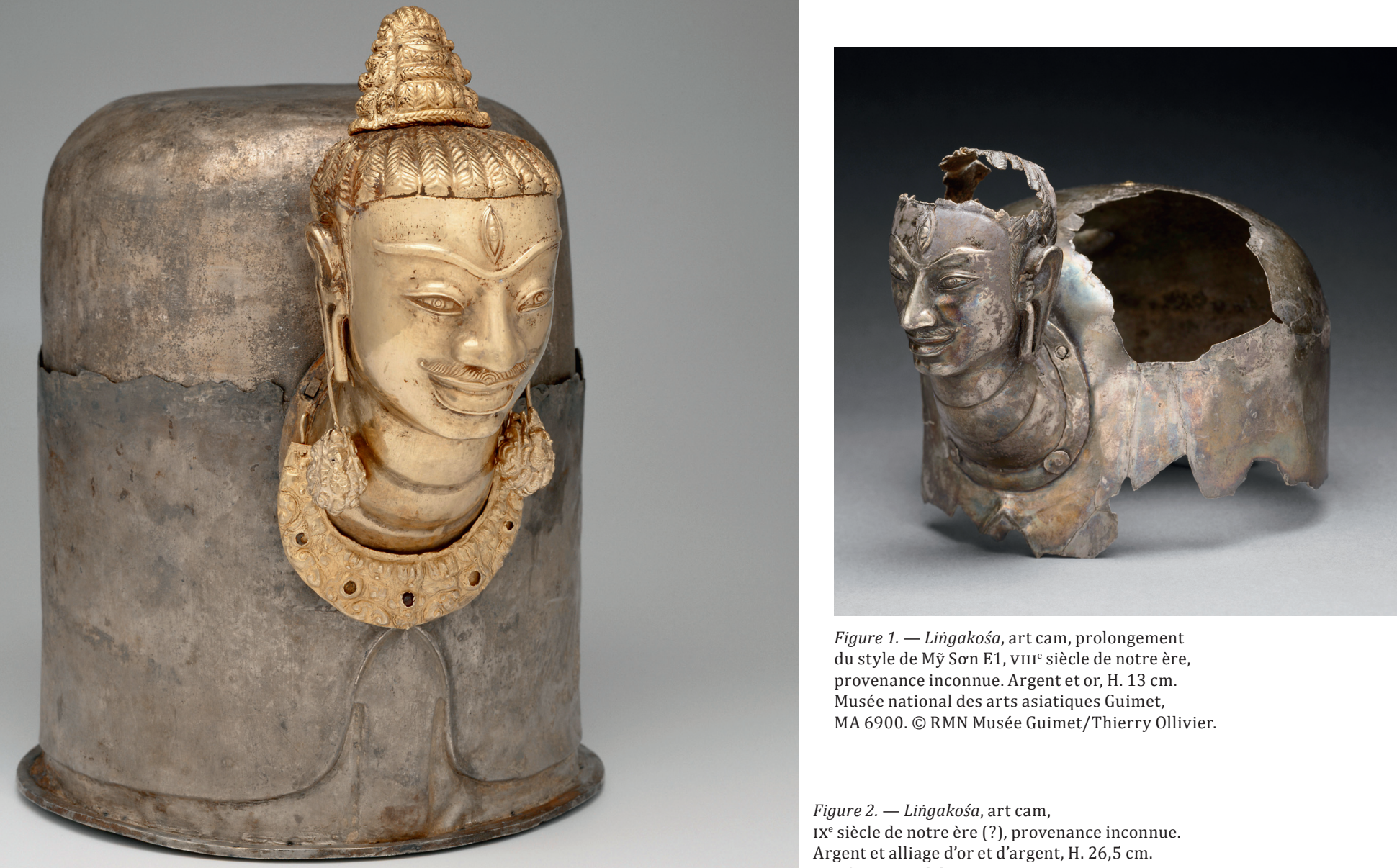

Figure 1. - Lingakośa, art cam, prolongement du style de Mỹ Sơn E1, viII ${ }^{\mathrm{e}}$ siècle de notre ère, provenance inconnue. Argent et or, $\mathrm{H} .13 \mathrm{~cm}$ Musée national des arts asiatiques Guimet, MA 6900. (C) RMN Musée Guimet/Thierry Ollivier.

Figure 2. - Lingakośa, art cam,

$\mathrm{IX}^{\mathrm{e}}$ siècle de notre ère (?), provenance inconnue. Argent et alliage d'or et d'argent, $H .26,5 \mathrm{~cm}$.

Musée national des arts asiatiques Guimet,

MA 6835. (C) RMN Musée Guimet/Thierry Ollivier.

Cette intuition fut ensuite confirmée par Louis Finot, qui s'appuyait sur la lecture d'inscriptions cames dans lesquelles cet objet est également mentionné, mais de façon plus précise que dans les inscriptions khmères. Dans la majorité des cas, L. Finot proposa de considérer les kośa des listes de biens comme des « enveloppes [de linga]». Il fit fond sur plusieurs références indiennes communiquées par A. Barth et publia des représentations de kośa indiens ${ }^{4}$. Fort de ce parallèle et constatant que nombre de ces objets étaient ornés de visages, L. Finotémit l'idée que « le rôle du kośa paraît avoir été de donner à la pierre symbolique la forme d'un dieu personnel ». Depuis ces découvertes, Kamaleswar Bhattacharya a consacré une étude plus complète à la question des kośa et a poursuivi les réflexions de L. Finot quant à la fonction de cette enveloppe dans la pensée śivaïte. Il proposait ainsi l'interprétation suivante: «[...] the

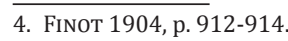

Śaivas, when they invented the custom of enclosing the linga in a sheath, had in mind this philosophical conception. - Śiva was conceived as a person with his linga (-śarīra) ["the subtle body"] enclosed in the kośa of his "gross body" »5.

La majorité des occurrences de kośa relevées dans l'épigraphie khmère, lorsqu'elles désignent cet objet particulier associé au rituel śivaïte, font vraisemblablement référence à une gaine en métal permettant de couvrir le linga ${ }^{6}$. En l'absence de précisions, il est impossible de spécifier, en outre, si celle-ci était ou non pourvue d'un visage comme c'est souvent le cas en Inde.

Le contexte doit donc, pour ces occurrences, nous aider à proposer la bonne identification. Ainsi, dans l'inscription K. 265 (piédroit nord, 1. 7 ; 881 śaka; IC IV, p. 102), ou dans l'inscription K. 1198 (face A,

\footnotetext{
5. BHATtACHARYA 1966, p. 7.

6. C'est le cas de dix-huit occurrences sur les trente-neuf que nous avons relevées.
}

l. 30 ; 936 śaka ; NIC II-III, p. 245, 251), le fait que ces occurrences correspondent à des objets explicitementaffectés à des linga nous oriente clairement vers ce sens technique.

Enfin, au-delà du sens symbolique souligné par K. Bhattacharya, il est intéressant de signaler que, du point de vue du rituel, cet objet est avant tout compris comme une parure - décrite par exemple dans le chapitre consacré aux ornements dans le Rauravāgama (Kp 43, 13-14a et 14b-15a; DAGENS \& BARAZER-BILLORET 2000 , p. $254-255)^{7}$ - et que cette interprétation avait apparemment été adoptée par les Khmers. En effet, comme nous pouvons le voir dans l'inscription K. 194, le suvarnakośa était mis au nombre des parures (thnim) de la divinité 8 .

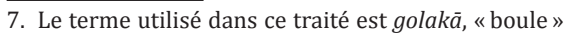
il n'est pas précisé qu'il doive être pourvu d'un visage. Bruno Dagens rappelle que la description de l'Ajitāgama est plus claire et indique seulement que cette parure doit être « comme le Linga » (ibid., n. 10).

8. K. 194, face A, l. 48-B. l. 3 ; 1041 śaka: dravya ti bhagavat pāda kamrateñ 'añ ta guru śrīdivākarapaṇdita jvan to 
Quels sont les éléments présents dans les inscriptions khmères concernant la description physique de cet objet?

Les matériaux dont ils sont composés sont relativement variés : les kośa peuvent être en or (suvarṇnakośa; K. 669, face C, l. 5-6; 894 śaka ; IC I, p. 169, 182), en argent (kośa prāk; K. 136, face c, l. 25 ; X śaka; IC VI, p. 285-286) ou en cuivre (kośa lañgau ; K. 450, l. 2 ; $\mathrm{X}^{\mathrm{e}}$ śaka ; IC III, p. 110, 112). Ces variations sont fonction de la richesse des donateurs ${ }^{9}$. Trois occurrences nous fournissent des informations concernant le poids de ces objets, respectivement 2 katți d'argent (K. 910, 1.14; 573 śaka; IC V, p. 39), 2 jyan் et 18 lin d'un matériau malheureusement non précisé (K. 258, face A, l. 38 ; $\mathrm{XI}^{\mathrm{e}}$ śaka; IC IV, p. 180, 195) et 1 jyan் d'argent (K. 108, l. 17 ; $\mathrm{x}^{\mathrm{e}}$ śaka; NIC II-III, p. 120). Ces mesures de poids sont difficiles à évaluer et variaient sans doute en fonction du lieu et de l'époque; ces données ne nous fournissent donc que peu d'informations ${ }^{10}$.

kamratenjagat śrīśikharīsvara thnim suvarnakośa makuta kundala keyūra \{2\} kañkana kaṇțhi udaravandha kāñci nūpura pāduka ta tām sarvvaratna ta gi ti vraḥ pāda kamrateñ 'añ śrīsūryyavarmmadeva 'oy dāna nā thve $\{3\}$ vrah dikșā..

« Biens que le vénérable K. 'A., le Guru Śrī Divākarapaṇdita a offerts au K. J. Śrī Śikharî́vara : parures : gaine en or, coiffe, pendants d'oreilles, brassards... bracelets, collier, ceinture de torse, ceinture, anneaux de chevilles, sandales avec toutes sortes de gemmes dessus, que S. M. Śrī Sūryavarmadeva (lui) avait données (à l'occasion de son) initiation... » (CCEDÈs \& DUPONT 1943, p. 143.)

Nous relevons également que cette liste de parures de Śiva correspond assez bien à l'ensemble des ornements des images exposés dans le chapitre 94 du Diptāgama: « Les parures des images sont: le diadème (pattta) large de 5 doigts et d'une longueur égale à la circonférence du Linga, la tiare, le couvre-chignon (karnamakuța?), le collier, les brassards, les bracelets, la ceinture de hanche (kațisūtra), les (couvre-)pieds ( $p a \bar{d} d a$ ?) et les anneaux de chevilles, la ceinture basse (udarabandha), la double-bandoulière et la ceinture, ainsi que les boucles d'oreilles et les fleurs pour les oreilles » (94.385-387ab; DAGENS, BARAZET-BILLORET \& LEFÈVRE 2009).

9. Elles illustrent bien l'introduction du chapitre $43 \mathrm{du}$ Rauravāgama: «Maintenant je présente brièvement les parures telles que la coiffe. Il faut (les) confectionner en or pur de la Jambū ou en or (ordinaire) ou en argent ou en cuivre en fonction des disponibilités financières » (DAGENS \& BARAZET-BILLORET 2000, p. 254).

10. SouTIF 2009, p. 143-152, 304. Sachant que 1 katti $=$ 1 jyan் $=20$ lin, en admettant que la valeur de ces mesures n'ait pas trop évolué, le poids de ces trois kośa varie du simple au triple - ou en tout cas du simple au double en ce qui concerne les deux kośa en argent - ce qui laisse supposer une certaine disparité de taille.

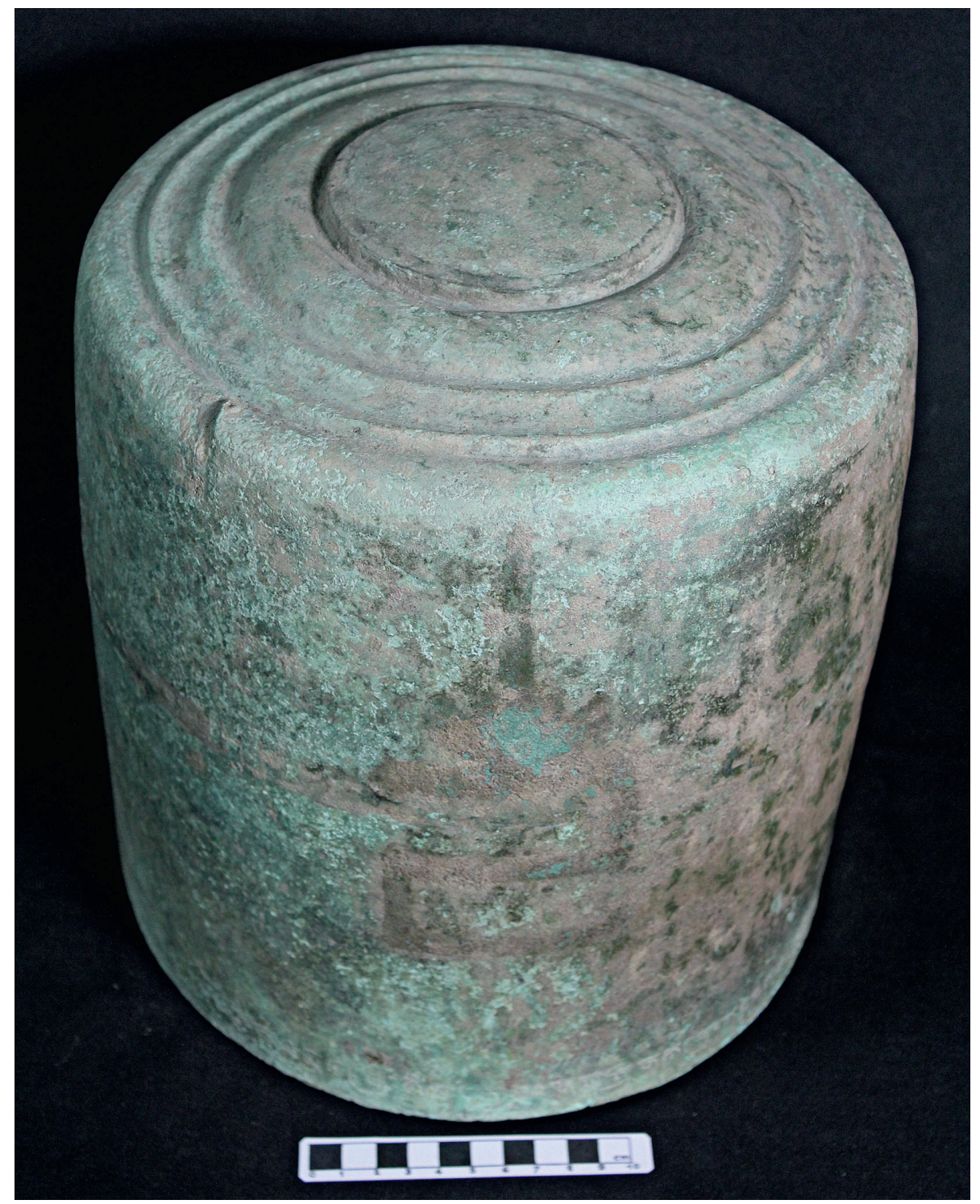

Figure 3. - Lingakośa, provenance inconnue. Bronze, H. $25 \mathrm{~cm}$; D. entre 25,2 et 29,5 cm. Musée national du Cambodge, ga 7304. Photo Chea Socheat.

Les inscriptions cames sont plus disertes que les inscriptions khmères, c'est par exemple le cas de l'inscription C. 86.1 de Mỹ Sơn qui rapporte que le roi cam Jaya Parameśvaravarman a donné un « kośa d'argent avec un visage d'or » à un linga (raupyakośa suvarṇnamukha; 1.7 ; XII ${ }^{\mathrm{e}}$ śaka; LEPOUTRE 2013, p. 264). Nous apprenons ainsi que ces gaines d'Asie du Sud-Est pouvaient, tout comme les gaines indiennes, être ornées d'un visage représentant la divinité, ce que l'on ne peut déduire des éléments présents dans les inscriptions khmères. L'épigraphie came témoigne également de kośa à quatre, cinq et même six faces, mises en relation avec les régions de l'espace ${ }^{11}$. Les matériaux cams sont également précieux concernant ce sujet puisque plusieurs gaines de linga cams ont été retrouvées. Les plus célèbres se trouvent au musée Guimet. Elles correspondent particulièrement bien à la description fournie par l'inscription C. 86.1 (fig. 1 et 2) ${ }^{12}$ et l'une d'entre elles résout, par l'exemple, l'incongruité

11. BHATTACHARYA 1966, p. 6; voir le passage cité intégralement dans l'article d'Anne-Valérie Schweyer, infra p. 108-109.

12. BAPTISTE 2001 , p. $112-113 ; 2002$ a et 2002 b, p. 158-159. 
que l'on pouvait voir dans l'offrande de pendants d'oreilles à un linga rapportée par l'inscription K. 194, puisqu'il s'agit précisément d'un kośa en argent à visage en or comme dans l'inscription C. 86.1 (cf.n. 8) ${ }^{13}$.

L'identification des kośa mentionnés dans les listes de biens en lien avec des linga comme gaine enchâssant celui-ci ne fait donc aucun doute, mais encore récemment, aucun exemple d'un tel objet n'était connu au Cambodge. Cette lacune fut comblée en 2009, quand un collectionneur fit don d'un lingakośa en bronze au Musée national du Cambodge à Phnom Penh. Il y est conservé sous le numéro ga 7304. Sa provenance est malheureusement inconnue (fig. 3 à 7).

Il s'agit d'un objet creux de forme cylindrique mesurant $25 \mathrm{~cm}$ de hauteur et fermé de façon convexe à l'une de ses extrémités. Du fait de déformations manifestes, le diamètre de ce cylindre varie entre 25,2 et $29,5 \mathrm{~cm}$. Son poids est de 2,6 kg. La pièce semble avoir été fondue en deux parties comme en atteste le raccord nettement visible entre celles-ci, leur bord étant crénelé pour en faciliter la fixation (fig. 4).

Elle comporte une inscription d'une ligne en khmer angkorien qui longe sa base. Cette inscription a été inventoriée par le programme de Corpus des inscriptions khmères sous le numéro K. 1286.

Présentons d'abord ce court texte avant de nous pencher plus avant sur le décor de ce kośa (fig. 5 et 7).

\section{Édition $^{14}$ :}

(0 1096 saka jamnvana vrah śivatejah tapasvi ta kamrateña jagata śrī sūryyapandita

\section{Traduction :}

«1096 śaka ${ }^{15}$, offrande du vénérable Śivatejạ̣, ascète, au Kamraten் Jagat Śrī Sūryapaṇdita. »

\footnotetext{
13. Un autre exemple de kośa cam en argent est présenté par Anne-Valérie Schweyer dans ce dossier (infra, p. 107113). Au sujet des kośa cams, $c f$. également GuY 2000, LoBo 2005, SCHWEYER 2008 et NGô 2008.

14. Édition fondée sur les photos EFEO CIK_K1286_1 à 7 ; cf. fig. 5.

15. Soit $1174 / 1175$ de notre ère. On notera qu'il s'agit d'une période charnière, bien que peu connue de l'histoire
}

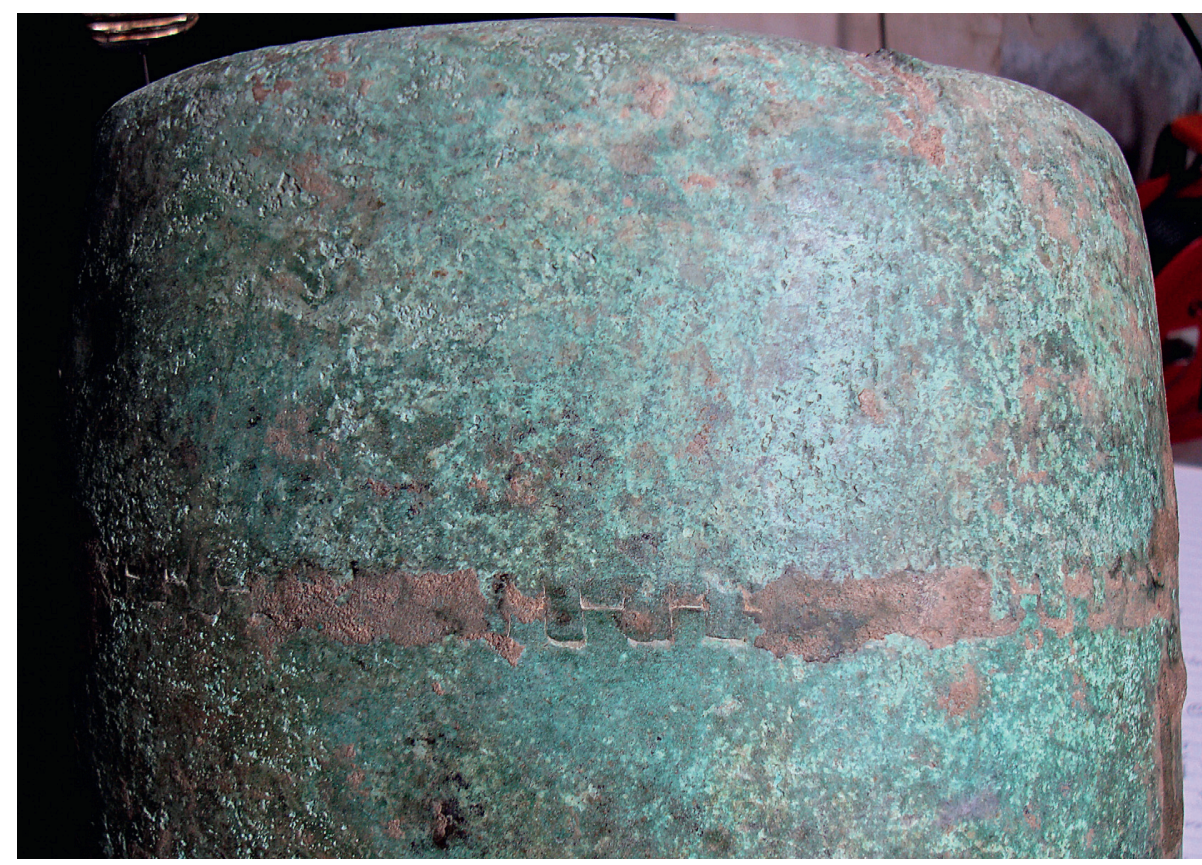

Figure 4. - Lingakośa du Musée national du Cambodge (cf. fig. 3). Détail de la fixation des deux parties du kośa. Photo D. Soutif.

Concernant la date de l'inscription et celle de l'objet, bien que l'écriture soit assez souple, certaines hésitations nous semblent devoir être interprétées comme des traces d'outil qui laissent supposer que le graveur a travaillé sur l'objet fini. Même s'il est vraisemblable que la gravure a été réalisée peu de temps après, l'inscription nous paraît postérieure à la réalisation de son support et ne permet donc pas de le dater précisément comme c'est parfois le cas lorsque les textes sont gravés dans la cire.

En ce qui concerne le texte proprement dit, il faut d'abord noter que, contrairement à la majorité des autres objets en métal inscrits connus, cette donation n'est pas le fait du roi, mais d'un certain Śivatejah ${ }^{16}$ qualifié

angkorienne, pour laquelle nous ne disposons que de très peu de documents. En effet, ce don fut effectué à la fin du règne de Tribhuvanādityavarman dont la carrière prit fin par une défaite contre les Cams et la prise d'Angkor, avant d'être suivie par la prise de pouvoir par Jayavarman VII. 16. Le composé śivatejah n'apparaît qu'une fois dans l'épigraphie khmère, dans l'inscription K. 380 : kāla vrạ śivateja hamraten jagat śrí śikhariśvara ta yal pratyakșa prādurbhāva, «... lorsque la sainte splendeur śivaïte du K. J. Śrī Śikharīśvara est apparue aux yeux dans une manifestation... » (piédroit ouest, l. 14-15 ; IC VI, p. 261). de tapasvi ${ }^{17}$. Malheureusement, son nom n'apparaît dans aucune autre inscription. S'il a pu faire un tel don, ce religieux, tout « ascète » qu'il fût, n'était pas déshérité ${ }^{18}$.

Il est vrai que, bien que l'on retrouve assez régulièrement des mentions d'ascètes dans les inscriptions khmères, leur statut n'est pas évident à préciser. Manifestement, il changeait en fonction de l'institution religieuse à laquelle ces ascètes appartenaient et de la fonction qu'ils y occupaient. Ainsi, il est vraisemblable que les dignitaires de haut rang qualifiés de vrah kamraten் 'añ tapasvi dans les inscriptions K. 207 (1. 46; 964 śaka; IC III, p. 18) et K. 879 (1. 6; 925 śaka; IC III, p. 236) avaient une position sociale plus élevée que les ascètes des

17. On trouve les orthographes tapasvi, tapassvi, tapaśvi et tapsvī dans les parties khmères des inscriptions (DAK, S. v., p. 182)

18. Notons que ce texte montre qu'au Cambodge, le don de kośa n'était pas l'apanage des rois comme les sources épigraphiques ont pu le laisser supposer en contexte cam À ce sujet, John Guy notait en effet: «These [Cham] inscriptions clearly link the kośa to ritual and magical functions central to the political legitimacy and survival of the ruler and his state » (GuY 2000, p. 51). Cf. également LOBо 2005, p. 88 et SCHWEYER 2008, p. 19. 


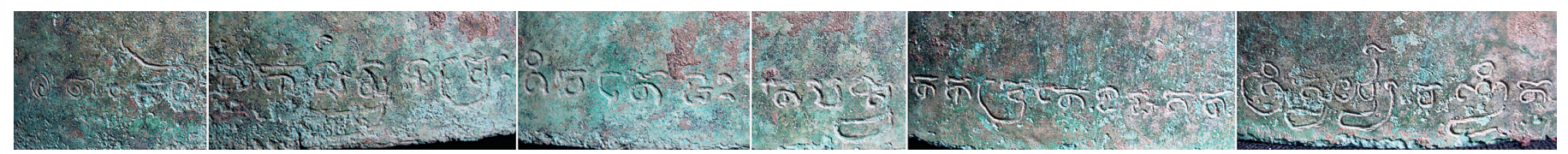

Figure 5. - Lingakośa du Musée national du Cambodge (cf. fig. 3). Inscription 1286, texte. Photos D. Soutif.

« résidences forestières » (tapovanāvāsa) évoqués dans l'inscription K. 410 (1. 10 ; 944 śaka; $R S$ II, p. 10-11), à moins que les tapasvi portant le titre de vrah kamraten' 'añ ne correspondent tout simplement à ceux qui dirigeaient des communautés d'ascètes, comme cela semble être le cas dans l'inscription K. 195. D’une manière générale, il semble que ces religieux pouvaient disposer de moyens puisqu'il est justement précisé dans ce dernier texte que des «ascètes de Śivasthāna » étaient à l'origine de la fondation d'un ãśrama qu'ils dotèrent d'esclaves «achetés avec leurs biens ». Il est mentionné, de plus, que ces tapasvi percevaient des honoraires (st. III ; 971 śaka ; IC VI, p. 249250). L'inscription K. 830 rapporte d'ailleurs qu'un de ces ascètes de Śivasthāna acheta une terre à grands frais (à savoir : plusieurs vases en métal, vingt bœufs et dix yau de vêtements) pour en faire don à une divinité (1. 2-5 ; 994 śaka ; IC V, p. 278).

Dans le même ordre d'idées, l'inscription K. 524 (1039 śaka ; IC III, p. 134135), rapporte que le vénérable ascète Vidyāvāsa (vrah tapasvi vidyāvāsa) reçut les honneurs de trois rois successifs et fonda trois linga sous le règne de Sūryavarman II. George Cœdès proposait de l'identifier à un Khloñ mentionné dans une inscription du Phnom Bayang dix ans plus tôt (K. 852, l. 6; 1029 śaka ; IC I, p. 267). De plus, celui-ci est désigné un an plus tard comme le Vrah Kamraten 'An் Ta Guru Vidyāvāsa dans l'inscription K. 523 (face D, l. 26 ; 1040 śaka ; IC III, p. 140). Nous sommes donc en présence d'un religieux de très haut rang et il n'est donc pas surprenant que des « ascètes » de ce type aient disposé d'une certaine fortune qu'ils pouvaient consacrer à des œuvres pies ${ }^{19}$.

19. Pour un cas probablement parallèle, voir l'article d'Arlo Griffiths et de Brice Vincent dans ce dossier.

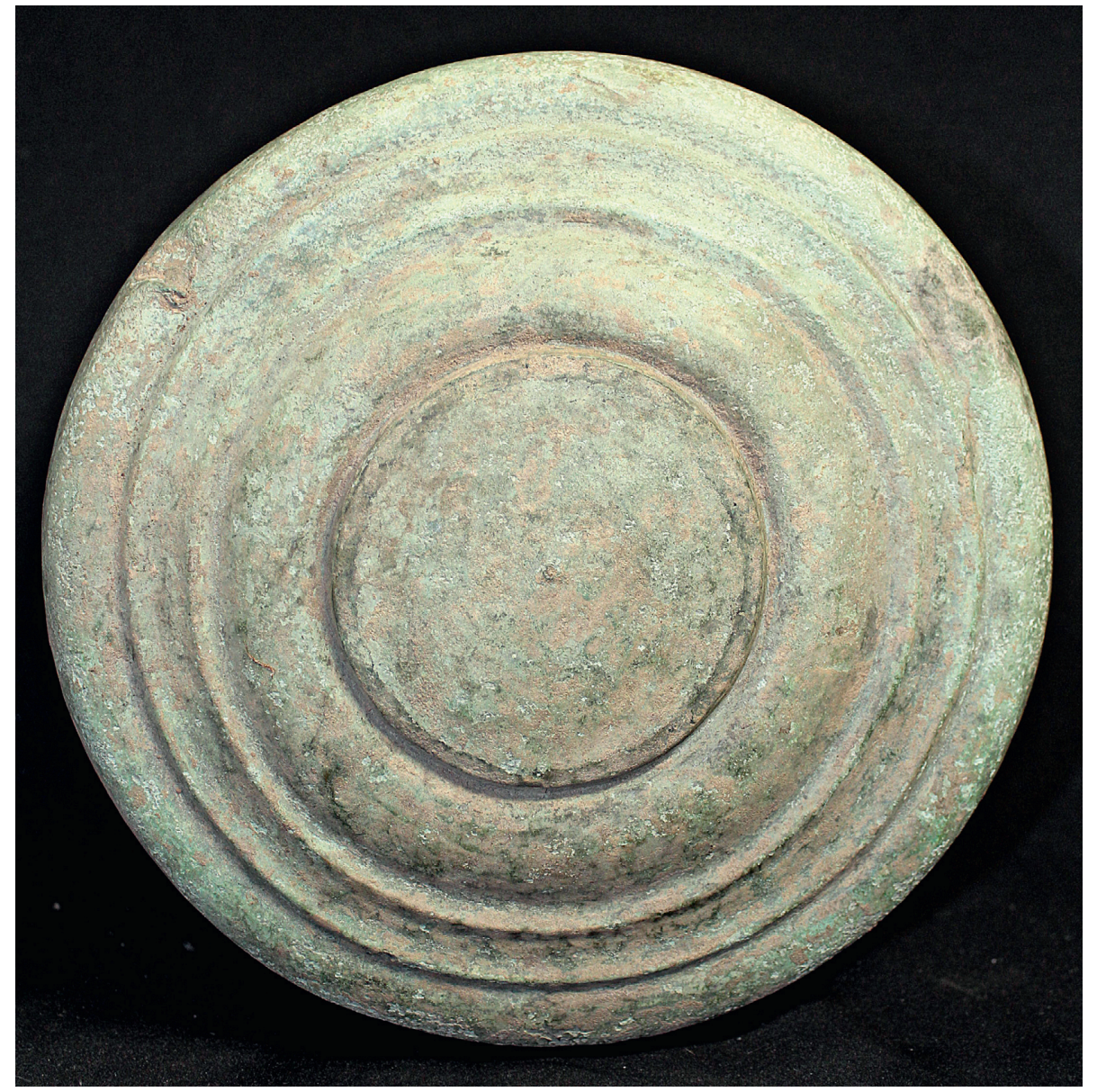

Figure 6. - Lingakośa du Musée national du Cambodge (cf. fig. 3). Détail de la moulure. Photo Chea Socheat.

Le nom de la divinité, Sūryapaṇịita, est également intéressant. Il faut d'abord signaler qu'il n'est pas attesté dans le corpus épigraphique comme nom de dieu. Il s'agit également d'un nom inhabituel pour une divinité, qui fait plutôt penser à un nom de dignitaire. On peut supposer que le nom de ce Kamraten Jagat est à rapprocher des troisième et cinquième catégories identifiées par G. Cœdès dans son étude des inscriptions des monuments de Jayavarman VII (CoEDÈs 1951, p. 99). La première de celles-ci correspondait aux statues dont les noms sont en rapport avec celui du fondateur; G. Cœdès prenait pour exemple la Kamraten Jagat Śrī Dharanīndreśvarī, l'une des divinités citées dans l'inscription K. 913, fondée à Ta Prohm par le Vrah Kamraten் 'Añ Śrī Dharanīndrapaṇdita (ibid., p. 104). 
La cinquième catégorie de G. Cœdès rassemblait les divinités qui sont l'image de personnages dont le nom entre dans la composition de celui de la divinité. C'était le cas cette fois, par exemple, du Kamraten Jagat Śrī Dharaṇindradeva de l'inscription K. 625, image (rūpa) du Vrah Kamraten் 'Añ Śrī Dharaṇindrapaṇdita installé au Preah Khan d'Angkor ${ }^{20}$ (ibid., p. 112).

Dans notre inscription K. 1286, il est difficile de trancher, et il est surprenant que le nom n'ait pas subi de transformation en prenant un suffixe en -iśvara ou -deva. Cependant, on connaît à ce jour un dignitaire nommé Sūryapanditita qui aurait pu être à l'origine du nom de ce dieu. Il est mentionné dans l'inscription K. 692 (st. L, LI, LVII, LVIII, LX ; 1117 śaka ; IC I, p. 237-238, 247-248). Cette inscription nous apprend que, dans ce cas, Sūryapanditita est un titre honorifique et que ce dignitaire portait auparavant ceux de Bhūpendrapaṇdita et de Rājendrapaṇdita. Il semble qu'il s'agissait d'un très haut personnage, que le roi récompensa par les attributs les plus prestigieux (st. LIII) et qui occupait les fonctions de «maître des conseillers, sacrificateur du koțihoma de son maître [le roi], maître des présidents de cour (...), arbitre dans les procès en matière religieuse et en matière profane » (st. LIV). Sachant que ce personnage, commémoré de façon posthume dans cette inscription datée de 1195 de notre ère, servait en réalité sous le règne de Sūryavarman II (1113ca. 1150 de notre ère), les dates pourraient correspondre. De plus, quand on sait que la stance LVII de cette même inscription nous apprend qu'il avait de son vivant érigé sa propre image avec celle de son épouse, par dévotion envers ses parents, et que nous ne lui connaissons pas d'homonyme, il semble raisonnable de penser que nous sommes en présence d'un don en faveur de l'image divinisée de ce haut dignitaire.

\footnotetext{
20. Comme l'avait remarqué G. Cœdès, il s'agit sans doute du fondateur de la divinité de Ta Prohm. Une seconde inscription de Preah Khan (K. 907) le mentionne à nouveau, mais il faut noter que le texte de K. 625 précise que cette image est cār klvān, expression signifiant " graver (ou ciseler) le corps, ou 'soi-même' » selon G. Cœedès, qui laisse supposer qu'il fit installer lui-même cette statue à son image (CCEDÈs 1951, p. 112, n. 2 ; cf. également DAK, s. v., p. 76).
}

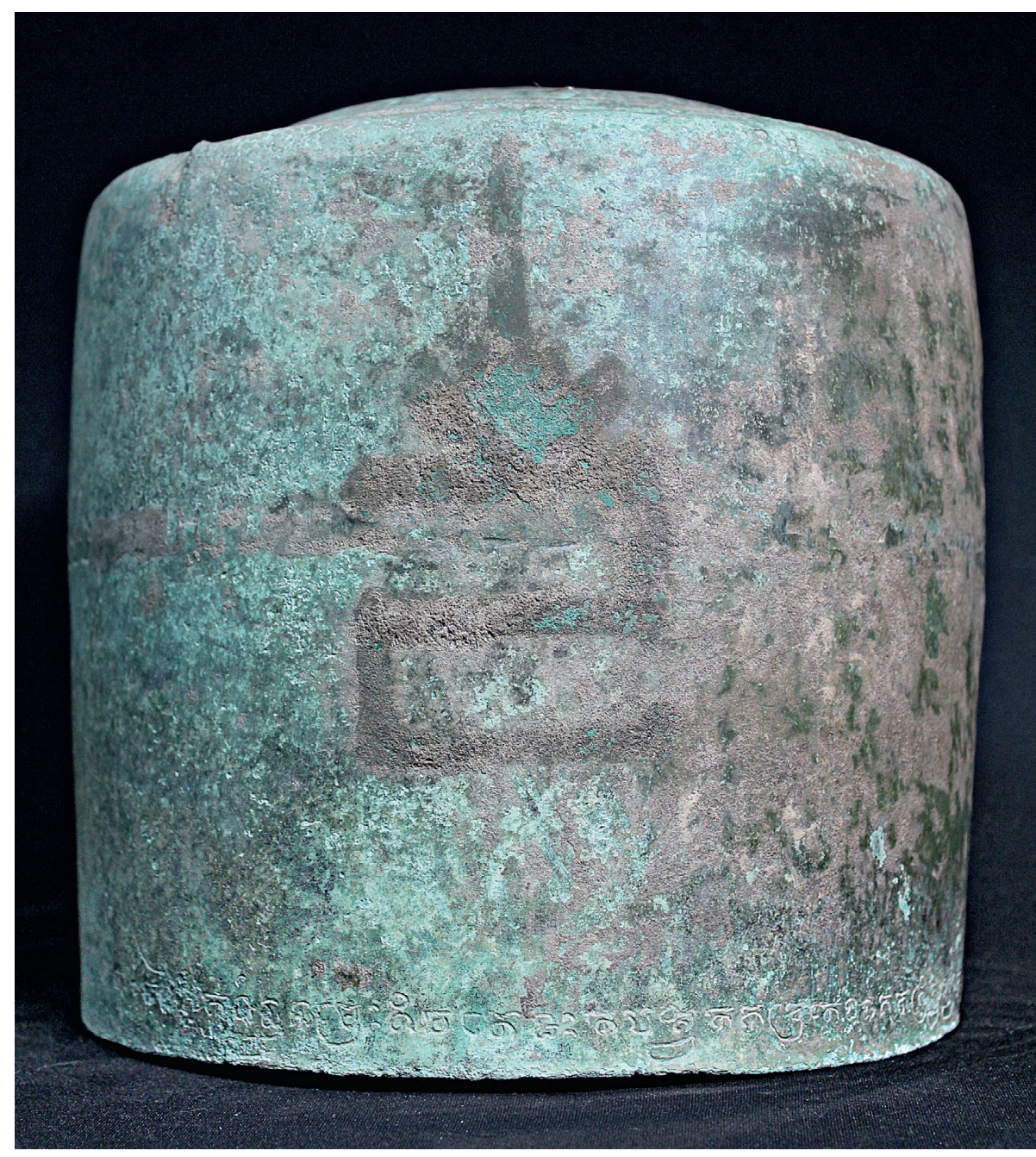

Figure 7. - Lingakośa du Musée national du Cambodge (cf. fig. 3). Inscription et vestiges de la fixation d'un décor en forme de syllabe om̆. Photo Chea Socheat.

Revenons au support de l'inscription. Le décor de ce lingakośa est très sobre. En dehors de l'inscription qui court le long de son bord inférieur, il se limite, à première vue, à un élément mouluré formant des cercles concentriques ornant sa partie supérieure (fig. 6). Il comportait pourtant un autre décor, aujourd'hui disparu. Au-dessus de l'inscription, on peut en effet observer une trace en forme de $o$ (2) surmontée du candrabindu (fig. 7). Ceci correspond sans doute aux restes d'une soudure corrodée ou à des résidus d'éléments organiques ${ }^{21}$ liés à un précédent collage qui fixait un signe om̆ sur le kośa.

21. Cette seconde hypothèse nous a été proposée par Huot Samnang, responsable de l'atelier de restauration des métaux du Musée national de Phnom Penh.
Cet élément permet de confirmer l'identification d'un autre objet mentionné dans les listes de biens destinés aux divinités. En effet, il est possible de relever dans ces énumérations quatre occurrences de dons de omkāra ou «syllabe $o \check{m} »^{22}$. Or, ces donations sont toutes dédiées à un linga de Siva ${ }^{23}$ et, dans deux cas, ces ornements sont mentionnés juste

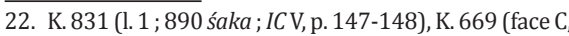
l. 5-6; 894 śaka; IC I, p. 169), K. 618 (l. 32 ; $\mathrm{x}^{\mathrm{e}}$ śaka; NIC II-III, p. 226-227) et K. 1198 (face A, l. 31 ; 931 śaka ; NIC II-III, p. 245, 251).

23. Le contexte des donations de l'inscription K. 618 n'est pas clair du fait des lacunes que présente le texte. Cependant, la mention du śivalinga du Temple des Brahmanes dans l'inscription K. 617 (1. 27-28; NIC II-III, p. 227) avec laquelle elle forme un groupe (incluant également les inscriptions K. 619 et K. 620), provenant toutes du temple de Sek Ta Tuy, nous incite à penser que le contexte de K. 618 était également śivaïte. 
après des kośa que nous avons identifiés précisément comme étant des gaines de linga (K. 669, K. 1198). G. Cœdès avait déjà supposé qu'il s'agissait d'un ornement spécifique du linga (IC I, p. 182, n. 2) et rappelait que l'on retrouve un don équivalent dans l'inscription came C. 86.2 de Mỹ Sơn (l. 5 ; 1152 śaka ; LEPoutre 2013, p. 265), que nous avons déjà évoquée à propos du lingakośa. Cependant, aucun élément probant n'était venu jusqu'ici confirmer cette hypothèse ${ }^{24}$. Grâce au lingakośa du Musée national du Cambodge, nous comprenons enfin le sens de cette donation et pouvons déterminer à quel objet il correspondait: la gaine était ornée de la syllabe sacrée om̆, qui lui était adjointe.

Le matériau dont elle était faite est spécifié dans trois des quatre occurrences : il s'agit d'or (mās, suvarṇa) dans les inscriptions K. 669 et K. 1198 et, plus curieusement, d'étain et de plomb (on்kāra tamirek trapu $)^{25}$ dans l'inscription K. 618. Les occurrences de K. 669 et K. 1198 nous apprennent également que ces ornements étaient parfois sertis de gemmes dont la nature n'est pas précisée ${ }^{26}$.

Il convient de relever que cet ornement n'était toutefois peut-être pas spécifiquement destiné au linga ou à son kośa. En effet, il n'est pas rare que les coiffures des divinités soient ornées de la syllabe sacrée om̆. Elle est parfois gravée (fig. 8) ${ }^{27}$,

24. Saveros Pou, par exemple, se demandait encore en 2001 (NIC II-III, p. 229, n. 4) : «Serait-ce donc un élément dans la constitution du linga en iconographie?»

25. L. Finot avait lu onkāra tel ta trapu (FInot 1928, p. 57), mais la lecture de S. Pou nous semble confirmée par l'examen de l'estampage EFEO n. 523, en dépit de la lecture incertaine de l'anusvära.

26. Dans l'inscription K. 669, face C, l. 5-6 (IC I, p. 169, 182) : nā vrah kamraten் 'añ śivalinga suvarṇnakośa I cumvala ta gĩ ońkāra I ratna ta gī I neh mās, « (parure) du V.K.'A. Śivalinga : 1 kośa en or avec une couronne, 1 syllabe om̆ avec 1 pierre dessus, tout cela en or». Dans l'inscription K. 1198, face A, l. 30-31 : vrah koșa hanira vrah suvarna'ońkāra mvāya thmo ta gi, «(un) saint kośa en hanir, une sainte syllabe om̆ en or, avec (une) pierre dessus »; S. Pou lit un 2 après ta gi mais note 3 gemmes dans sa traduction; il nous semble qu'il n'y a pas de chiffre (estampage EFEO n. 1654 ; NIC II-III, p. 245, 251).

27. Lors de l'évaluation de cet article, l'un des relecteurs nous a fait remarquer que «l'adjonction d'un om̌ a parfois été utilisée pour 'śivaïser' des Avalokiteśvara à la période post-Jayavarman VII », l'image du Buddha Amitābha étant alors bûchée et le om̆ sculpté à son emplacement. L'aspect mais on ne peut écarter l'hypothèse de parures mobiles. À notre connaissance, le seul objet retrouvé pouvant s'apparenter à un ornement de ce type en métal est une plaque de bronze découpée de façon à former la syllabe om̆, retrouvée au Bakong $^{28}$ (fig. 9). On peut imaginer que celui qui était accolé à notre lingakośa était similaire à cette plaque, si ce n'est qu'au lieu d'être pourvu d'un tenon de fixation, il avait été simplement collé ou soudé.

Nous voyons ainsi que cette gaine de linga conservée au Musée national du Cambodge constitue un objet remarquable par plusieurs aspects, permettant d'éclairer des termes apparaissant régulièrement dans l'épigraphie et prouve, si besoin était, l'intérêt qu'il y a à étudier conjointement le corpus épigraphique et le mobilier archéologique. La perte du contexte de sa découverte est d'autant plus regrettable. Elle montre donc, encore une fois, la nécessité de sensibiliser les collectionneurs à l'intérêt historique et non pas seulement esthétique que peuvent revêtir les pièces dont ils font l'acquisition.

Julia Estève, College of Religious Studies, Mahidol (Thaïlande) esteve.julia@yahoo.fr

Dominique Soutif, EFEO (Siem Reap) dominique.soutif@efeo.net

rrégulier de la pierre au niveau du om̆ de l'Avalokiteśvara du musée Guimet présenté ici en figure 8 pourrait en effet témoigner d'une telle transformation et rend cette hypothèse très vraisemblable.

28. Cet objet a été exhumé dans un bâtiment annexe du Bakong situé près du mur oriental de la première enceinte, au sud du gopura. Il était placé dans un vase qui contenait également des bagues (JFCA XV, p. 63; RCA Bakong, octobre 1939).

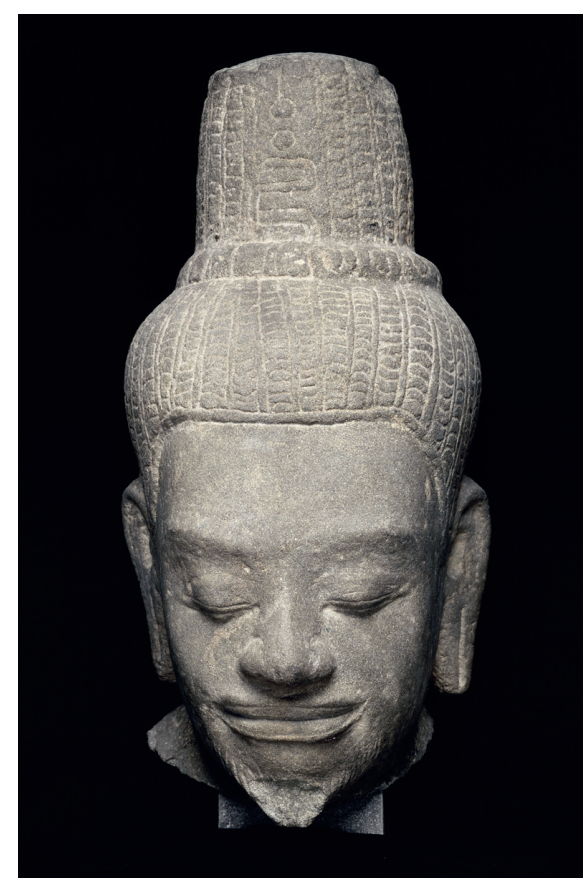

Figure 8. - Tête de Śiva (?), $\mathrm{XII}^{\mathrm{e}}-\mathrm{XIII}^{\mathrm{e}}$ siècle de notre ère, provenance inconnue. Grès, H. $36 \mathrm{~cm}$. Musée national des arts asiatiques Guimet, MG 14895. (C) RMN Musée Guimet/Michel Urtado.

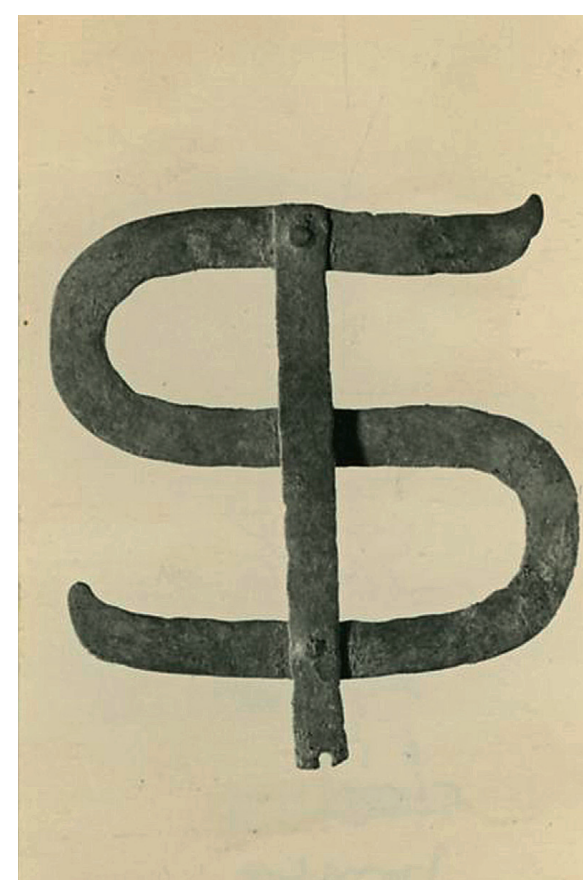

Figure 9. - Syllabe om̆ découpée dans une plaque en bronze et fixée sur une barre par des rivets, Bakong, datation inconnue. H. $6,2 \mathrm{~cm}$; L. 5,8 cm. Photo EFEO représentant le dos de l'objet placé à l'envers. 
Annexe 1 : occurrences de kośa dans l'épigraphie khmère

\begin{tabular}{|c|c|c|c|c|c|c|}
\hline Occurrence & $\begin{array}{l}\text { Ortho- } \\
\text { graphe }\end{array}$ & $\begin{array}{c}\text { Date } \\
\text { (ère śaka) }\end{array}$ & $\begin{array}{l}\text { Contexte } \\
\text { religieux de } \\
\text { l'occurrence }\end{array}$ & Identification & Bibliographie & $\begin{array}{l}\text { Détails sur l'objet } \\
\text { et composés }\end{array}$ \\
\hline K. 55, st. VIII & kośa & $\mathrm{VI}^{\mathrm{e}}$ & śivaïte & trésor & $I S C$, p. 57-59 & $\begin{array}{l}\text { jeu de mots sur kośa = trésor } \\
\text { et kośa = objet de culte; } \\
\text { il s'agit vraisemblablement } \\
\text { d'1 [linga]kośa }\end{array}$ \\
\hline K. 80 , st. I & $k o s ̦ a^{1}$ & $\mathrm{VI}^{\mathrm{e}}$ & śivaïte & lingakośa & $I S C$, p. $45-46$ & - \\
\hline K. $125,1.11$ & kośa & 923 & śivaïte & lingakośa & $\begin{array}{l}\text { CoEDÈs 1906, } \\
\text { p. } 142 \\
\end{array}$ & - \\
\hline K. $136,1.25$ & kośa & $\mathrm{x}^{\mathrm{e}}$ & śivaïte $^{2}$ & lingakośa & IC VI, p. 285 & 1 kośa en argent \\
\hline K. 162 , st. XX & kośa & $\mathrm{IX}^{\mathrm{e}}$ & śivaïte & lingakośa & IC VI, p. 105 & 1 kośa en or \\
\hline $\begin{array}{l}\text { K. } 256 \text {, piédroit III, } \\
\text { l. } 14\end{array}$ & kośa & $\mathrm{IX}^{\mathrm{e}}$ & $?$ & coupe (à libation) & \begin{tabular}{|l} 
COEDÈs \& \\
DuPONT 1937, \\
p. 391 \\
\end{tabular} & yajñakośa \\
\hline K. 258, face A, l. 38 & koșaṇa & 1018 & śivaïte & lingakośa & $\begin{array}{l}\text { IC V, p. 180, 195, } \\
\text { n. } 5\end{array}$ & $\begin{array}{l}2 \text { saints koṣaṇa [pesant] } 2 \text { jyan, } \\
18 \text { lin }\end{array}$ \\
\hline $\begin{array}{l}\text { K. } 262 \text {, piédroit nord, } \\
\text { l. } 10\end{array}$ & kośa & 890 & vishnuite? & coupe (à libation) & IC IV, p. 110 & yajñakośa \\
\hline K. 263 , face D, l. 12 & kośa & 890 & vishnuite? & coupe (à libation) & IC IV, p. 127 & yajñakośa \\
\hline K. $450,1.2$ & kośa & $\mathrm{x}^{\mathrm{e}}$ & śivaïte & lingakośa & IC III, p. 110 & 1 kośa en cuivre \\
\hline K. 485, st. LXXVIII & kośa & $\mathrm{XII}^{\mathrm{e}}$ & sans objet & trésor & IC II, p. 170 & - \\
\hline K. 485, st. LXXXII & kośa & $\mathrm{XII}^{\mathrm{e}}$ & bouddhique & lampadaire & IC II, p. 171 & dìpakośa \\
\hline K. 485, st. LXXXIV & kośa & $\mathrm{XII}^{\mathrm{e}}$ & bouddhique & $?$ & IC II, p. 171 & - \\
\hline K. $660,1.2$ & kośa & 963 & sans objet & $\begin{array}{l}\text { couvercle } \\
\text { d'aiguière }\end{array}$ & IC I, p. 196 & - \\
\hline K. 669, face C, l. 6 & kośa & 894 & śivaïte & lingakośa & IC I, p. 169 & 1 kośa en or (suvarṇakośa) \\
\hline
\end{tabular}

1. Dans le composé ākhandalānamrakirītakoșah, qu'A. Barth traduit par: (la victoire est au dieu) «qui repose sur la tiare altière d'Ākhandala » et note à propos de ce composé: " [Ākhanḍala =]Indra. Le texte dit: "qui a la tiare... pour kosha", c'est-à-dire : qui est porté par elle, comme une fleur est portée par sa capsule. On peut aussi décomposer ānamra, "inclinée (devant lui)" ». Une fois identifiée la nature du kośa en tant que parure de Śiva, il est assez naturel de penser que c'est bien dans ce sens qu'il est utilisé et que Śiva a la tiare d'Indra pour kośa.

2. Destiné au Kamrateñ Jagat Śrī Vrahmarakșa 'Āy Rudrā, sans doute une divinité śivaïte. 


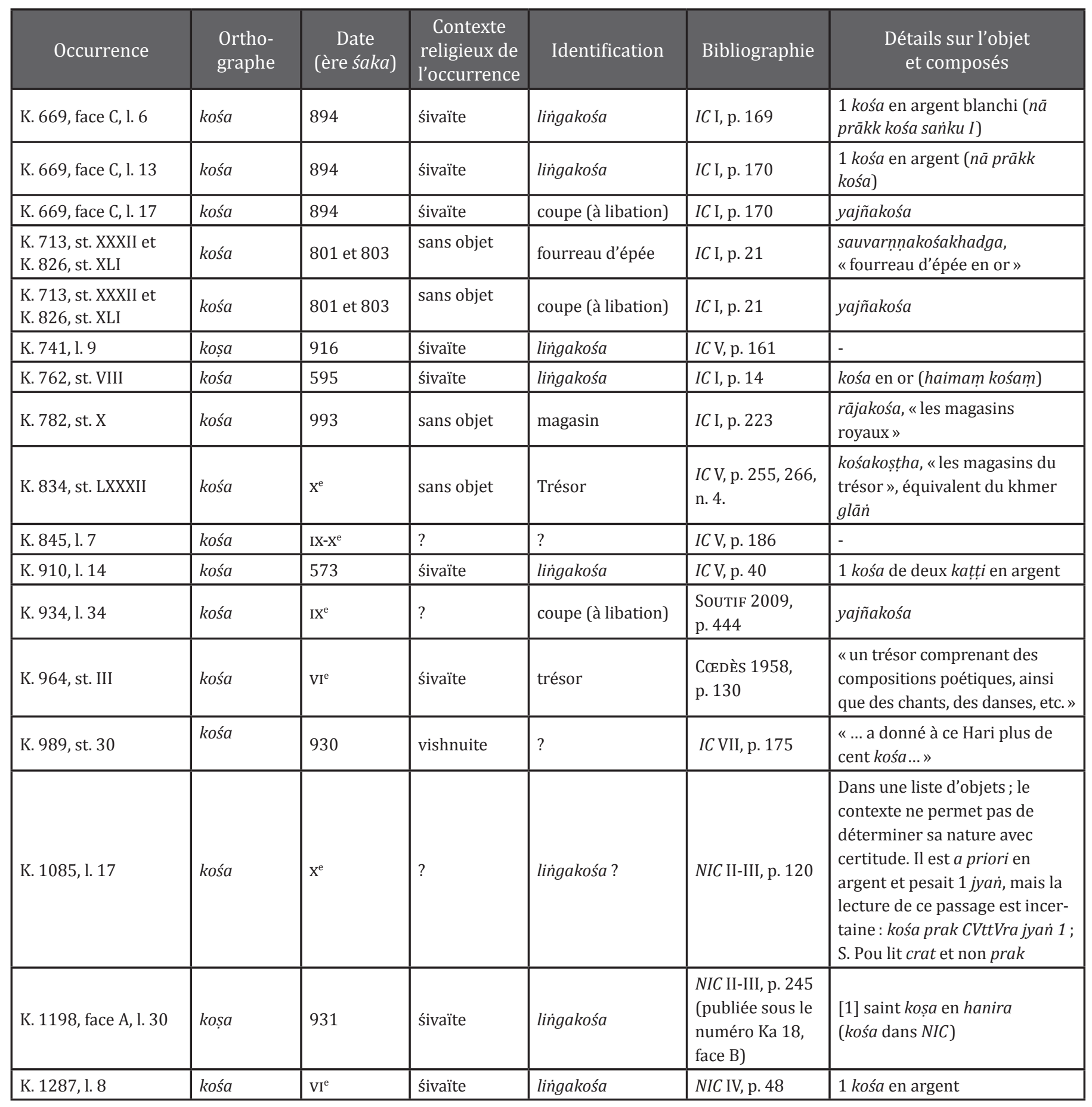




\section{Abréviations}

APK: Articles sur le pays khmer; cf. CœED̀̀s 1989/1992. BEFEO : Bulletin de l'École française d'Extrême-Orient. DAK: Dictionary of Angkorian Khmer; cf. JENNER 2009. DSF : Dictionnaire sanskrit-français; $c f$. STCHOUPAK, NITT \& RENOU 1932

IC: Inscriptions du Cambodge ; cf. CCEDÈs 1937-1966. ISC: Inscriptions sanscrites du Cambodge; cf. BARTH 1885 ISCC: Inscriptions sanscrites de Campā et du Cambodge; cf. BERGAIGNE 1893.

JFCA: Journaux de fouilles de la Conservation d'Angkor.

NIC: Nouvelles inscriptions du Cambodge; cf. Pou 2001 et 2011.

RCA : Rapports de la Conservation d'Angkor.

RS : Recueil des inscriptions du Siam; cf. CCEDÈs 1929.

\section{Bibliographie}

BAPTISTE Pierre, 2001: «Activités du musée national des Arts asiatiques-Guimet: Asie du Sud-Est», Arts Asiatiques, 56, p. 112-115.

- 2002a : «Un étui couvre-linga au musée des Arts asiatiques-Guimet », Revue du Louvre et des Musées de France, 3, p. 13-17.

- 2002b: «Activités du musée national des Arts asiatiques-Guimet : Asie du Sud-Est », Arts Asiatiques, 57, p. 157-162.

BARTH Auguste, 1885 : Inscriptions sanscrites du Cambodge, tiré des notices et extraits des manuscrits de la Bibliothèque nationale et autres bibliothèques, tome XXVII, $1^{\text {re }}$ partie, $1^{\text {er }}$ fascicule, Paris, Imprimerie nationale, p. 1-180.

BERgAIgne Abel, 1893 : Inscriptions sanscrites de Campä et $d u$ Cambodge, tiré des notices et extraits des manuscrits de la Bibliothèque nationale et autres bibliothèques, tome XXVII, $1^{\text {re }}$ partie, $2^{\mathrm{e}}$ fascicule Paris, Imprimerie nationale, p. 181-632.

BHATTACHARYA Kamaleswar, 1966: «Linga-Kośa », Artibus Asiae Supplementum, 23, Essays Offered to G. H. Luce by His Colleagues and Friends in Honour of his Seventy-Fifth Birthday, vol. II, p. 6-13.

BRunNer-LACHAUx Hélène, 1963-1998 : Somaśambhupaddhati, texte, traduction et notes par $\mathrm{H}$. BrunnerLachaux, 4 vol., Pondichéry, Institut français d'indologie/EFEO [vol. I : Rituel quotidien, 1963; vol. II : Rituels occasionnels (I), 1968; vol. III : Rituels occasionnels (II), 1977; vol. IV : Rituels optionnels, 1998].
CoEdÈs George, 1906: «La stèle de Ta Prohm », BEFEO 6(1-2), p. 44-82. [Réimpr. 1992, APK, vol. 2, p. 11-49.]

- 1929: Recueil des inscriptions du Siam, 2. Inscriptions de Dvāravatī, de Çrīvijaya et de Lăvo, Bangkok, Bangkok Times Press.

- 1937-1966: Inscriptions du Cambodge, 8 vol., Hanoi \& Paris, EFEO (Collections de textes et documents sur l'Indochine, 3).

- 1951: «Études Cambodgiennes, 39. L'épigraphie des monuments de Jayavarman VII », BEFEO, 44 p. 97-119. [Réimpr. 1989, APK, vol. 1, p. 343-366].

- 1958: «Nouvelles données épigraphiques sur l’histoire de l'Indochine Centrale», Journal Asiatique, 246, p. 125-142.

- 1989/1992 : Articles sur le pays khmer, 2 vol., Paris EFEO. [Réimpr. des articles sur le Cambodge parus dans le BEFEO et dans les Cahiers de l'École française d'Extrême-Orient.]

CoEDÈs George \& DUPONT Pierre, 1937: « Les inscriptions de Pràsàt Kôk Pô », BEFEO, 37, p. 379-413. [Réimpr. 1992, $A P K$, vol. 2, p. 81-98].

— 1943 : «Les stèles de Sdŏk Kăk Thom, Phnom Sandak et Práh Vihār », BEFEO, 43, p. 56-134. [Réimpr. 1992 $A P K$, vol. 2, p. 167-265.]

DAGENS Bruno \& BARAZER-BILLORET Marie-Luce, 2000 Le Rauravāgama: un traité de rituel et de doctrine śivaïte, Pondichéry, Institut français de Pondichéry.

DAGENS Bruno, BARAZER-BILloRET Marie-Luce \& LEFÈVRE Vincent, 2009: Dīptāgama, édition critique, tome III, avec la collaboration de S. Sambandha Sivacarya et de Christèle Barois, Pondichéry, Institut français de Pondichéry.

FINOT Louis, 1904 : « Notes d'épigraphie, XI. Les inscriptions de Mĩ-sơn », BEFEO, 4(4), p. 897-977.

GRIFFITHS Arlo, 2005: "La stèle d'installation de Śrī Tribhuvaneśvara: une nouvelle inscription préangkorienne du Musée national de Phnom Penh (K. 1214) », avec la collaboration de Gerdi Gersch heimer et J. C. Eade, Journal Asiatique, 293(1), p. 11-43.

GuY John, 2000: «The Kośa Masks of Champa: New Evidence », in Loво Wibke et Reimann Stephanie (éd.), Southeast Asian Archaeology, 1998, Proceedings of the 7th International Conference of the European Association of Southeast Asian Archaeologists, Berlin, 31 August-4 September 1998, Hull, Centre for Southeast Asian Studies, University of Hull, Berlin, p. 51-60.

JACQUes Claude, 1970 : «Études d'épigraphie cambodgienne, IV. Deux inscriptions du Phnom Bakhen (K. 464 et K. 568); V. La stèle du Prasat Cha Chuk (K. 1034) », BEFEO, 57, p. 57-89.
JENNER Philip N., 2009: A Dictionary of Angkorian Khmer Canberra, Pacific Linguistics.

LEPOUTRE Amandine, 2013 : «Étude du corpus des inscriptions du Campā, IV. Les inscriptions du temple de Svayamutpanna : contribution à l'histoire des relations entre les pouvoirs cam et khmer (de la fin du XII ${ }^{\mathrm{e}}$ siècle au début du XIII ${ }^{\mathrm{e}}$ siècle) ", Journa Asiatique, 301(1), p. 205-278.

Lово Wibke, 2005 : «'Linga' et 'kośa' au Champa. Culte et iconographie», in BAPTISTE Pierre \& ZÉPHIR Thierry (dir.), Trésors d'art du Vietnam. La sculpture du Champa, Paris, Réunion des musées nationaux, p. 88-95.

Monier-Williams Monier, 1899: A Sanskrit-English Dictionary Etymologically and Philologically Arranged with Special Reference to Cognate IndoEuropean Languages, Oxford, Clarendon Press. [rééd. 1990, Delhi, $10^{\mathrm{e}}$ tirage.]

NGô Văn Doanh, 2008: « Kośa in the Religion and Art of Champā », in JELEN János et al., Gold Treasures of the Cham Kingdoms from the Collection of Dr. Zelnik, vol. 2, Budapest, JelNet Ltd., p. 44-47.

Pou Saveros, 2001 : Nouvelles inscriptions du Cambodge tomes II et III [en un seul volume], Paris, EFEO.

- 2004 : Dictionnaire vieux khmer-français-anglais. An Old Khmer-French-English Dictionary. Vacanānukram khmaer cas'-paramn-angles, Paris, L'Harmattan, $2^{\mathrm{e}}$ édition. [1 ${ }^{\mathrm{e} r e}$ éd. $1992+$ supplément.]

- 2011: Nouvelles inscriptions du Cambodge, tome IV, Paris, L'Harmattan.

SCHWEyer Anne-Valérie, 2008: «Kośa in Cham Inscriptions: Political Power and Ritual Practices », in JELEN János et al., Gold Treasures of the Cham Kingdoms from the Collection of Dr. Zelnik, vol. 2, Budapest, JelNet Ltd., p. 13-27.

SoutiF Dominique, 2009: «Organisation religieuse et profane du temple khmer du VII ${ }^{\mathrm{e}}$ au XIII ${ }^{\mathrm{e}}$ siècle», 3 vol., Thèse de doctorat, Paris, université Sorbonne nouvelle-Paris 3.

Stchoupak Nadine, Nitti Luigia \& Renou Louis, 1932: Dictionnaire sanskrit-français, Paris, Adrien Maisonneuve. [Rééd. 1990, $6^{\text {e }}$ tirage.] 